\title{
On-line Optimal Ranging Sensor Deployment for Robotic Exploration
}

This paper was downloaded from TechRxiv (https://www.techrxiv.org).

\section{LICENSE}

CC BY-NC-SA 4.0

SUBMISSION DATE / POSTED DATE

28-07-2021 / 02-08-2021

CITATION

Santoro, Luca; Brunelli, Davide; fontanelli, daniele (2021): On-line Optimal Ranging Sensor Deployment for Robotic Exploration. TechRxiv. Preprint. https://doi.org/10.36227/techrxiv.15066513.v1

DOI

10.36227/techrxiv.15066513.v1 


\title{
On-line Optimal Ranging Sensor Deployment for Robotic Exploration
}

\author{
Luca Santoro, Davide Brunelli, Senior Member, IEEE, and Daniele Fontanelli, Senior Member, IEEE
}

\begin{abstract}
Navigation in an unknown environment without any preexisting positioning infrastructure has always been hard for mobile robots. This paper presents a self-deployable ultra wideband UWB infrastructure by mobile agents, that permits a dynamic placement and runtime extension of UWB anchors infrastructure while the robot explores the new environment. We provide a detailed analysis of the uncertainty of the positioning system while the UWB infrastructure grows. Moreover, we developed a genetic algorithm that minimizes the deployment of new anchors, saving energy and resources on the mobile robot and maximizing the time of the mission. Although the presented approach is general for any class of mobile system, we run simulations and experiments with indoor drones. Results demonstrate that maximum positioning uncertainty is always controlled under the user's threshold, using the Geometric Dilution of Precision (GDoP).
\end{abstract}

Index Terms_-Unmanned autonomous vehicles, Positioning System, Robot sensing systems

\section{INTRODUCTION}

Mobile robotics, either terrestrial or aerial, have quickly registered incremental advances and interests from the industry and research community. Nowadays, they are pervasively applied in a variety of applications. Exploration of unknown environments has attracted an increasing attention due to their large application scenarios, such as search and rescue missions [1], disaster recovery [2], planetary exploration [3], photogrammetry [4], aerial inspection and monitoring of buildings and structures [5] [6], agriculture [7] and predictive maintenance [8]. Localisation and positioning capabilities are primary features for any autonomous exploration system. According to the application scenarios, several solutions can be used. The capability of positioning in an absolute reference system, usually with the GPS signal [9], is one of the most used techniques. However, many robot exploration activities are in GNSS-denied environments, such as indoor. In such challenging cases, alternative positioning methods are usually considered, e.g., visual-SLAM [10] [11], laser scanners [12]. Some of these techniques require non-negligible computing resources, work preferably in information-rich environment, and cannot guarantee a maximum target uncertainty (e.g., SLAM) [13]. Others, instead, have limited computational burden and can compute positioning under controlled uncertainty. Nevertheless, this class of solutions usually requires instrumented infrastructure in the surrounding with active or passive markers. Examples of this category are Radio Frequency (RF) active beacons for Radio Signal Strength Identification

\footnotetext{
Authors are with Dept. of industrial Engineering, University of Trento, Trento. (e-mail: \{luca.santoro, davide.brunelli, daniele.fontanelli\}@unitn.it).
}

(RSSI) [14] or Ultra Wide Band (UWB) [15] [16], while for passive solutions we can mention visual markers [17] or passive RFID tags [18]. The nature of the technology and the sensors embedded into the environment determine uncertainty during the exploration.

Providing positioning measurements with limited uncertainty for autonomous robot navigation is hard if an absolute reference as the GPS is not available. The achievable positioning performance depends both on the specific technology used by the sensing devices and on the algorithm defined for the placement of such devices [19]. When RF ranging sensors are considered, two different approaches are usually implemented to achieve the optimal placement in an unknown environment:

1) Off-line. The environment is analyzed, e.g., using statistics about the navigation paths, and the placement positions are determined to guarantee the desired target uncertainty. For example, in [20], three off-line algorithms are assessed and compared to find the candidate points of an additional beacon that maximizes the accuracy of the localisation service over the entire region.

2) Online. In this case, the environment may not be known upfront, and the anchors are deployed on-demand, e.g., when the localisation uncertainty approaches the maximum tolerable value. For instance, in [21], the robots use two different strategies to place a new sensor in the environment: measure the average of RSSI, and place the new sensor when this value falls under a predetermined value or based on a fixed distance.

Recently, ultra wideband UWB signals technology has been quickly confirmed as an effective and cheap solution for positioning problems. Different works are developed us- 
ing UWB to decrease the target uncertainty. For example, in [22] [23] [24], Kalman filters are used to fuse UWB and Inertial Measurement Units (IMU) for improving the position estimation and mitigating the problem of Non-Line-of-Sight (NLoS). In [25], the poor estimation along the vertical axis, which is a known weakness of the UWB infrastructures, is mitigated using inter-vehicle distance. In [26], a combination of LiDAR (Light Detection and Ranging) and UWB is investigated, exploiting LiDAR information to improve the UWB results.

The main characteristic of UWB technology is to use message exchange between mobile and fixed nodes. Typically, the mobile nodes are mounted on the robot's chassis, while the fixed nodes, named anchors, build up an infrastructure with known geometric characteristics. The fixed structure of nodes is usually deployed before starting any operations in the environment [27], hence adopting an off-line placement procedure. Although it seems simple, off-line placement is time-consuming and critical because any fault in this phase or anchor position uncertainty seriously influences the positioning system's precision.

To overcome infrastructure setup inaccuracies and provide a positioning system also for unstructured environments, where off-line analysis is inapplicable, we developed a method for dynamic placement and runtime extension of the infrastructure anchors. In our work, while exploring the environment, the mobile robot deploys new anchors to strengthen the infrastructures. Thus the ranging sensors are self-deployable and will extend the positioning reference at runtime during the robot exploration. Notice that this marks a striking difference with respect to the known literature. Indeed, existing solutions, e.g., [28], [29], cannot change the nodes infrastructure at runtime based on robot needs nor can adequately leverage the ratio of information versus uncertainty that a new added anchor injects in the multilateration problem. Moreover, our solution is robotcentered: existing solutions usually try to optimize the entire region as a whole, with evident computational burden issues and difficulties in unknown or partially known environments, while our solution is extremely light in terms of computing power and can be computed onboard the vehicle while it explores the (possibly unknown) environments. In particular, our solution proposes an online-incremental algorithm based on a genetic approach to solve the constrained optimization problem, which finds the most convenient placement for new anchors and reduces the number of deployments. The algorithm keeps the maximum target uncertainty below the user requirement, which is based on the Geometric Dilution of Precision (GDoP). It has to be noted that the proposed solution works with any metric able to express the positioning uncertainty, but the GDoP comes handy for this purpose [30].

In this work, we focus on positioning accuracy problems and not strictly on robot localisation, which requires the analysis of the problem's observability and the model of the robot dynamics [31]. We present how to use the proposed approach for a generic class of robot dynamics (e.g., ground or aerial vehicles), mainly focusing on positioning uncertainty.

The paper is organised as follows. Section II describes the placement metric adopted and the formulation of the
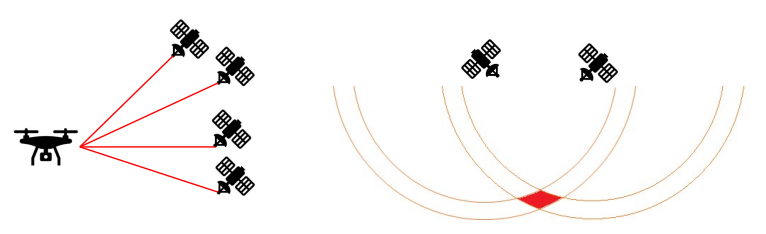

(a)
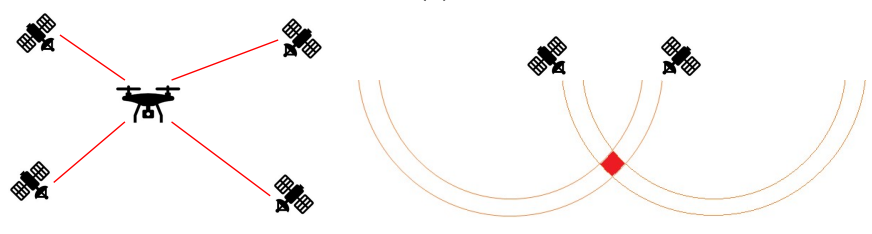

(b)

Fig. 1: Circular sector intersections from (a) bad or (b) good distributions of satellites

problem. Section III presents the solution based on a genetic algorithm to optimize the chosen metric and the optimal deployment manoeuvres, while Section IV investigates the position uncertainty of the proposed deployment algorithm. Section $\mathrm{V}$ discuss the simulation and experimental results. Finally, Section VI concludes the article and proposes future research directions.

\section{BACKGROUND}

GDoP is a metric adopted to quantify the precision and accuracy of the data received from GPS satellites, which is now being adopted to the wider set of generic positioning system [32], [33]. This metric indicates how well the satellites are geometrically organized. The lower the value, the better is the position accuracy [34]. A graphical representation of a poor or good geometric configuration is given in Figure 1.

GDoP is proportional to the ratio between the range error and position error [35], thus it is inversely proportional to the volume formed by the vectors from user to satellites and the number of satellites. Given the distance $\rho_{i}$ from the $i$-th anchor and assuming that all the ranging measurements have the same finite variance (hence, the homoscedastic property is satisfied), we define the variance associated to the ranging as $\sigma_{\rho}^{2}$ and, from [36], the covariance matrix of the positioning error is

$$
C=\sigma_{\rho}^{2}\left[\begin{array}{cccc}
\sigma_{x x}^{2} & \sigma_{x y}^{2} & \sigma_{x z}^{2} & \sigma_{x t}^{2} \\
\sigma_{y x}^{2} & \sigma_{y y}^{2} & \sigma_{y z}^{2} & \sigma_{y t}^{2} \\
\sigma_{z x}^{2} & \sigma_{z y}^{2} & \sigma_{z z}^{2} & \sigma_{z t}^{2} \\
\sigma_{t x}^{2} & \sigma_{t y}^{2} & \sigma_{t z}^{2} & \sigma_{t t}^{2}
\end{array}\right],
$$

where $\sigma_{\rho}^{2} \sigma_{x x}^{2}, \sigma_{\rho}^{2} \sigma_{y y}^{2}, \sigma_{\rho}^{2} \sigma_{z z}^{2}$ represent the variance of the estimated location along the corresponding axes and $\sigma_{t t}^{2}$ is the time offset of the receiver. Sub-metrics can be defined from (1) by adopting the trace on different sub-matrices, such as

$$
\left\{\begin{array}{l}
\mathrm{HDoP}=\sqrt{\sigma_{x x}^{2}+\sigma_{y y}^{2}}, \\
\mathrm{VDoP}=\sigma_{z z}^{2}, \\
\mathrm{PDoP}=\sqrt{\sigma_{x x}^{2}+\sigma_{y y}^{2}+\sigma_{z z}^{2}}, \\
\mathrm{GDoP}=\sqrt{\sigma_{x x}^{2}+\sigma_{y y}^{2}+\sigma_{z z}^{2}+\sigma_{t t}^{2}},
\end{array}\right.
$$




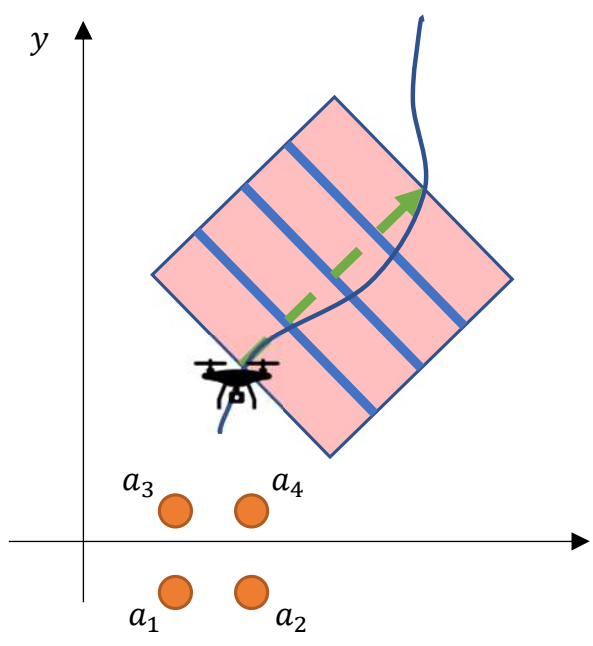

Fig. 2: A graphical representation of the problem statement. Example of subareas $S(i+(j-1) r / n, j r / n)$, with $\mathrm{j}=1, \ldots, \mathrm{n}$, using the described simplified approach.

where HDoP, VDoP and PDoP are the Horizontal, Vertical and Position Dilution of Precision, respectively, all derived from the GDoP. We moved these metrics to UWB infrastructures. Thus the position estimate of a receiver (called tag) in a generic three-dimensional space requires at least four UWB devices (called anchors). In contrast to (1), the time $t$ is not of interest for UWB ranging system because the propagation time of the signal is directly used for the time-of-flight measurement [37], hence the last column of $C$ will be neglected. Consequently, the PDoP metric in (2) is used in place of the GDoP.

\section{A. Problem formulation}

The contribution of this paper is to derive an optimal self-deployment on-line solution for UWB anchors during exploration problems and using the PDoP metric to define the target uncertainty. The optimal reduction of positioning uncertainties is tailored to the robotic platforms' requirements, saving onboard hardware and computation resources and time. The algorithm calculates the minimum number of anchors to deploy during the mission to accomplish the robot goal. More formally, let us consider the situation depicted in Figure 2. We assume that the anchors can only be deployed on the $X_{w} \times Y_{w}$ plane of the right-handed reference frame $\langle W\rangle=$ $\left\{X_{w}, Y_{w}, Z_{w}\right\}$, since the altitude of the placement is assumed to be not controllable. Notice that assuming no knowledge about the environment, we consider the worst possible conditions for the $z$ coordinates, i.e., that the multilateration algorithm is applied using coplanar anchors (flat terrain), hence we are assuming very poor VDoP. We denote with $a_{i}=\left[X_{i}, Y_{i}\right]^{T}$ the known coordinates of the anchor in $\langle W\rangle$ and projected on the plane $X_{w} \times Y_{w}$. Moreover, given $\mathcal{A}_{k}$ the set of all the anchors $a_{i}$, we define with $\overline{\mathcal{A}}_{k, n}$ as the set of all the combinations of $n$ anchors in $\mathcal{A}_{k}$. Therefore, $\mathcal{D}_{k}(s) \in \overline{\mathcal{A}}_{k, 4}$ denote the set of 4 anchors attaining the minimum value of PDoP in a certain position $s$. As a consequence, given:
- A sampling time $T_{s}$, which is induced by the sampling time of the available anchors;

- A planned exploration path $S_{p}=\left\{q_{i}\right\}_{i=1}^{h}$ is a set of $h$ viapoints on the plane, i.e. $q_{i}=\left[x_{q_{i}}, y_{q_{i}}\right]^{T}$;

- The actual position of the robot $s_{k}=\left[x_{k}, y_{k}\right]^{T}$ at time $k T_{s}$, supposed to be projected on the plane $X \times Y$, while $S_{k}=\left\{s_{i}\right\}_{i=0}^{k}$;

- An initial set $\mathcal{A}_{0}$ of 4 anchors that are in communication with the robot;

- A set of the overall deployed anchors $\mathcal{A}_{k}$ up to time $k T_{s}$;

- A maximum tolerable value $p^{m}$ of the PDoP along the exploration path;

- A maximum distance $\rho^{m}$ from an anchor to retrieve the ranging measurement;

- A PDoP function $g\left(\mathcal{D}_{k}\left(s_{k}\right), s_{k}\right)$ computed on the position $s_{k}$ given the anchors $a_{i} \in \mathcal{D}_{k}$;

the goal is to guarantee the existence of at least four anchors $\mathcal{D}_{k} \subset \mathcal{A}_{k}$ at time $k T_{s}$, such that the UWB positioning system can provide a PDoP $g\left(\mathcal{D}_{k}\left(s_{k}\right), s_{k}\right) \leq p^{m}, \forall s_{k}$ during the exploration while using the ranging data $\rho_{i, k}=\left\|s_{k}-a_{i}\right\| \leq$ $\rho^{m}$. To this extend, we define two problems:

i) the first is the Optimal placement problem (OPP)

$$
\begin{aligned}
& \min \# \mathcal{A}_{k} \text { s.t. } \\
& \exists \mathcal{D}_{k}\left(q_{i}\right) \subseteq \mathcal{A}_{k} \text { with } g\left(\mathcal{D}_{k}\left(q_{i}\right), q_{i}\right)<p^{m}, \forall q_{i} \in S_{p},
\end{aligned}
$$

where of course $\# \mathcal{A}_{k}$ are the number of elements in $\mathcal{A}_{k}$.

ii) The second, named Optimal Exploration and Placement Problem (OEPP), is based on OPP and defined on the actual robot positions $s_{k}$, instead of the planned positions $q_{i}$. The difference between the two problems is that OPP refers to the nominal robot trajectory, while OEPP considers all the maneuvers needed to deploy the new anchors.

In this paper, we will make explicit reference to a particular class of robots, namely Unmanned Aerial Vehicles (UAVs), even though the solution remains of general validity. It is worthwhile to mention that the algorithm is totally agnostic about the planner used to synthesize the robot path. One of the most used exploration methods is the sampling-based algorithm such as RRT [38]. Moreover, notice that the robot should entirely cover the exploration path at least once, i.e. $\exists k \in \mathbb{N}$ such that $s_{k}=q_{i}, \forall q_{i} \in S_{p}$.

\section{Anchors Deployment Algorithm}

At a first glance, OPP may appear a trivial problem that could be solved by computing $g\left(\mathcal{D}_{k}\left(s_{k}\right), s_{k}\right)$ at time $k T_{s}$ for the positions $s_{k}=q_{i}$ and place a new set of 4 anchors on the same pattern of Figure 2, when either $g\left(\mathcal{D}_{k}\left(s_{k}\right), s_{k}\right)=p^{m}$ or $\rho_{i, k}=\rho^{m}$ for some $a_{i} \in \mathcal{A}_{k}$. Then the robot starts over.

However, we observe three different problems with this approach (which is inspired by [39] applied to ground wheeled vehicles):

1) While placing 4 anchors satisfies the sufficient requirement for positioning, it does not guarantee that it is the only possible deployment.

2) When the UAV places the 4 anchors, the PDoP should be kept under control on the placement trajectory as well, hence a PDoP threshold $p^{\star}<p^{m}$ should be considered; 


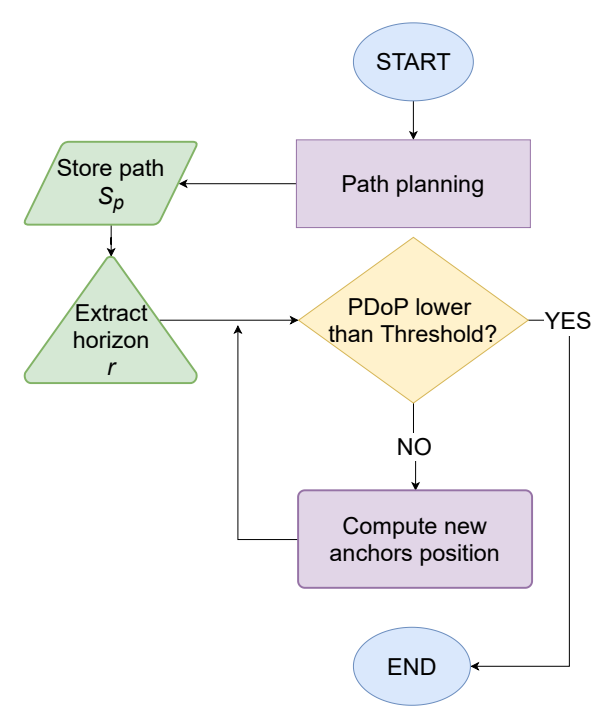

Fig. 3: Flowchart of GANP algorithm.

3) The next location to place the anchors may be a function of the future exploration path viapoints $S_{p}$, thus making some locations more favorable than others.

To address these issues, we propose the Genetic Anchor Node Placement (GANP) algorithm, which comprises a prediction of the PDoP function along the future path positions with a finite horizon $r$ and then compute the most favourable locations using a Genetic Algorithm (GA). More precisely, let us consider the robot is in position $s_{k}=q_{i}$, the algorithm starts by evaluating if $\exists q_{j} \in\left\{q_{i}, q_{i+1}, \ldots, q_{i+r}\right\}=S_{p}^{(i, r)} \subset S_{p}$ such that $g\left(\mathcal{D}_{k}\left(q_{j}\right), q_{j}\right)>p^{m}$. In such a case, we need to add at least one anchor. We can then grow a region $\mathcal{S}_{p}^{(i, r)}$ around the path portion $S_{p}^{(i, r)}$ of width $w$ by simply taking the local perpendicular to the path of length $w$ passing through each position $q_{j}=S_{p}^{(i, r)}$. However, to avoid a placement that concentrates the anchors in nearby positions, we split the finite horizon $r$ in $n$ subsets of $r / n$ points and then we could define non-overlapping regions $\mathcal{S}_{p}^{(i, r / n)}, \ldots, \mathcal{S}_{p}^{(i+r-r / n, i+r)}$ each hosting at most one new anchor. To simplify the subareas splitting, we simply take the line joining $q_{i}$ and $q_{i+r}$ and consider it as an approximation of the path, thus simplifying the searching regions comprised in $\mathcal{S}_{p}^{(i, r)}$ as sketch in Figure 2. Of course, the width $w$ of the searching region, the forecasting horizon $r$, and the number of sub-paths $n$ plays a crucial role in the algorithm performance, hence a tuning procedure is presented in Section V.

A flowchart of the GANP algorithm is depicted in Figure 3. Whenever one or more anchors should be placed according to the PDoP function, a list of the possible deployment coordinates is computed. The main idea is to allow at most one anchor in each subarea $\mathcal{S}_{p}^{(i+(j-1) r / n, j r / n)}$, with $j=$ $1, \ldots, n$. Suppose four anchors result placed in the $j$-th area to achieve an optimal PDoP value. In that case, it means that the considered $j$-th area is too detached (i.e., far) from the infrastructure, then $j-1$-th area is considered forcing the algorithm to generates at least one additional anchor.

The GA fitness function considers the optimal PDoP values for each point $q_{j} \in S_{p}^{i, r}$ by determining the set $\mathcal{D}_{k}\left(q_{j}\right)$. The PDoP quantities are then stored into a list and weighted according to the distance from $q_{i}$ : the more $\left\|q_{j}-q_{i}\right\|$ is larger, the higher is the weight. These values are then summed up and constitute the objective function to minimize. Notice that the weighting mechanism pushes the new possible anchors deeper along the exploration path. The constraint function of the GA imposes limitations to possible placement locations, which should be inside the subarea considered. Moreover, it must satisfy the PDoP limit $p^{m}$ along the path and minimize the PDoP along the considered horizon $r$. Notice that the GANP algorithm ensures the optimality of the OPP only. To extend the results to the OEPP, the nature of the deploying maneuver should be taken into account.

\section{A. Deploying Manoeuvres}

The GANP algorithm ensures that the value of PDoP never exceeds the maximum target value $p^{m}$. In fact, the algorithm governs the UAV controlled behaviour based on three states of a Finite State Machine. The UAV starts in Mission State (MS) where it follows the exploration path. When the condition is violated on the path horizon $r$, the optimal position of the anchor is determined, and the UAV switches to the Deployment State (DS). The UAV stores the last point reached along the mission path, say $q_{i} \in S_{p}$, and follows the shortest path towards the deploying location. After the anchor is positioned, the UAV either continues on the placement (if convenient, as described in the rest of this section) or it switches to the Placed State (PS). Here, a return-path to $q_{i}$ is generated and followed. When the robot reaches $q_{i}$ either switches back to DS (if additional anchors should be placed, or returns to MS, where the exploration continues. This motion pattern is pursued until the last point of the mission is reach, where the UAV decides which action to perform:

- Landing (or stopping) and becoming an integral part of UWB positioning infrastructure with its tag that switches to an anchor. This action can be fired by the battery level when it falls below a certain threshold;

- Continue the exploration mission, selecting a new exploration area with a new synthesized path and executing the described process;

- Alternatively, the robot can move back to the starting position, increasing the accuracy of the placed anchors.

The flowchart of the depicted algorithm is reported in Figure 4.

The path followed in the DS and PS states is crucial for any vehicle autonomy, especially when UAVs are considered. Therefore, the maneuver should take the shortest. While the placement path for a single anchor is straightforward, i.e., it is sufficient to move along the local perpendicular segment with respect to the planned path (see Figure 5a), the placement of more than one anchor may be tricky. Let us consider the case of Figure 5a: the robot may depart along the shortest path for each anchor to deploy, hence it stores $q_{i} \in S_{p}$, switches to DS, deploys the new anchor, switch to PS and goes back to $q_{i}$. Once $q_{i}$ is reached, it switches back to the DS state, moves towards $q_{j} \in S_{p}$, stores it and then moves to the next anchor 


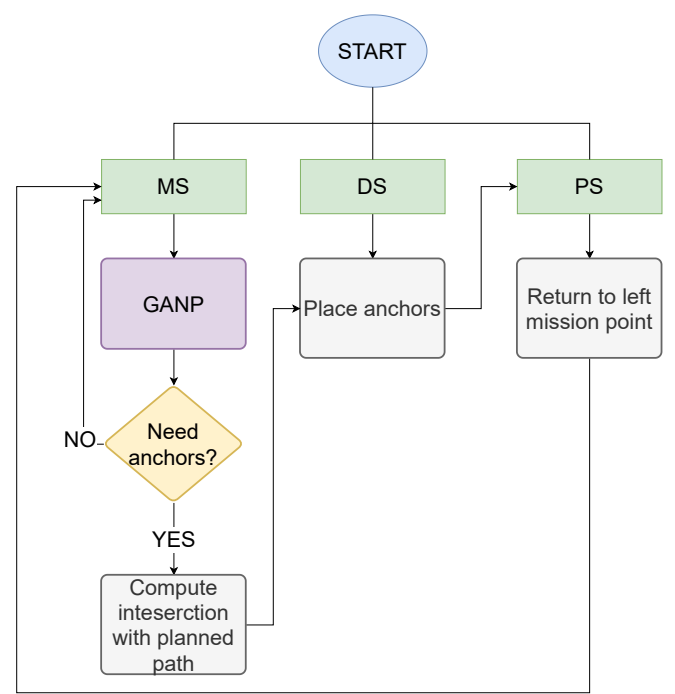

Fig. 4: Overall logic that govern the behaviour of the drone during its mission.

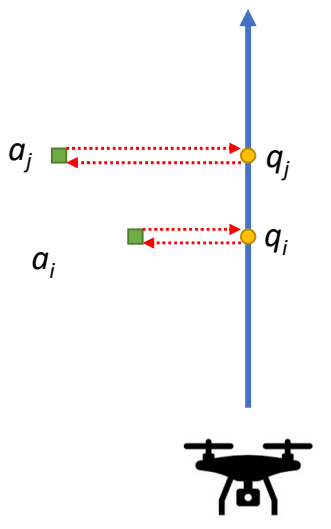

(a) Deployment path generation, each anchor has its relative intersections point.

Fig. 5: Deployment manoeuvres followed in DS and PS.

location. After the deployment, the UAV switches to the PS state, reaches $q_{j}$ and moves to the MS state to restart the exploration. However, this sequence may not be the shortest to place both anchors. To this end, it is evaluated if a shortest path exists by simply comparing the perimeter of standard geometric shapes (recall, that at most 4 anchors should be placed at once), with the constraint that the manoeuvre in DS starts and ends in the same point $q_{i}$ on the path to entirely cover the exploration path, as stated previously. For example, the path followed in DS for Figure $5 \mathrm{~b}$ is shorter than the path of Figure 5a, thus it will be selected.

It is now evident that, by embedding this manoeuvre generation in the constraint function of the GA, the OEPP problem is solved requiring the limit of the PDoP to be satisfied along the shortest deployment manoeuvres.

\section{UNCERTAINTY ANALYSIS}

In this section, we first present the explicit derivation of the PDoP function $g\left(\mathcal{D}_{k}\left(s_{k}\right), s_{k}\right)$ and an analysis of the positioning uncertainty accounting for the incorrect anchor deployment.

\section{A. Position Dilution of Precision}

As described in Section II, the PDoP function used in this paper $g\left(\mathcal{D}_{k}\left(s_{k}\right), s_{k}\right)$ is a function of the anchor locations $\mathcal{D}_{k}\left(s_{k}\right)=\left\{a_{i_{1}}, \ldots, a_{i_{m}}\right\}$ and of the point $s_{k}$ considered. In particular, defining with

$$
P=\left[\begin{array}{cc}
\frac{x_{k}-X_{i_{1}}}{\rho_{i_{1}}, k} & \frac{y_{k}-Y_{i_{1}}}{\rho_{i_{1}}, k} \\
\vdots & \vdots \\
\frac{x_{k}-X_{i_{m}}}{\rho_{i_{m}, k}} & \frac{y_{k}-Y_{i_{m}}}{\rho_{i_{m}, k}}
\end{array}\right],
$$

the Jacobian of the ranging function (4) and denoting with $Q$ the covariance matrix of the positioning error

$$
Q=\sigma_{\rho}^{2}\left(P^{T} P\right)^{-1}=\sigma_{\rho}^{2}\left[\begin{array}{cc}
\sigma_{x x}^{2} & \sigma_{x y}^{2} \\
\sigma_{y x}^{2} & \sigma_{y y}^{2}
\end{array}\right]
$$

the PDoP function $g\left(\mathcal{D}_{k}\left(s_{k}\right), s_{k}\right)=\sqrt{\sigma_{x x^{2}}+\sigma_{y y}{ }^{2}}$.

\section{B. Anchor deployment uncertainty}

The position of the UAV is computed using multilateration on distance measurements. The ranging measurements are collected by means of an UWB infrastructure, using a Single Side Two-Way-Ranging (SS-TWR) communication protocol. Assuming $n$ UWB anchors in known positions $a_{i}=\left[X_{i}, Y_{i}\right]^{T}$, $i=1, \ldots, n$, the ranging measurement from the $i$-th anchor at time $k T_{s}$ is defined as

$$
\bar{\rho}_{i, k}=\rho_{i, k}+\epsilon_{i, k}=\sqrt{\left(x_{k}-X_{i}\right)^{2}+\left(y_{k}-Y_{i}\right)^{2}}+\epsilon_{i, k},
$$

where $\epsilon_{i, k}$ is the ranging measurement uncertainty, usually considered as a white sequence with zero mean and variance $\sigma_{\rho}^{2}$ for all the anchors. Computing the difference of the squares of the distances $\Delta_{i j, k}=\bar{\rho}_{i, k}^{2}-\bar{\rho}_{j, k}^{2}$ from at least three anchors and using the same solution reported in [40], it is possible to derive the robot position estimates using a Weighted Least Squares (WLS) solution as

$$
\hat{s}_{k}=\left[\begin{array}{l}
\hat{x} \\
\hat{y}
\end{array}\right]=\frac{1}{2}\left(A^{(n)^{T}} N_{k}^{(n)^{-1}} A^{(n)}\right)^{-1} A^{(n)^{T}} N_{k}^{(n)^{-1}} h_{k}^{(n)},
$$

where $h_{k}^{(n)}$ is the vector of the indirect measurements $\Delta_{i j, k}$ and anchor positions, $A^{(n)}$ is a matrix containing the known anchor positions, while

$$
N_{k}^{(n)}=\sigma_{\rho}^{2}\left[\begin{array}{cccc}
\rho_{1, k}^{2}+\rho_{2, k}^{2} & \rho_{1, k}^{2} & \cdots & \rho_{1, k}^{2} \\
\rho_{1, k}^{2} & \rho_{1, k}^{2}+\rho_{3, k}^{2} & \cdots & \rho_{1, k}^{2} \\
\vdots & \vdots & \ddots & \vdots \\
\rho_{1, k}^{2} & \rho_{1, k}^{2} & \cdots & \rho_{1, k}^{2}+\rho_{n, k}^{2}
\end{array}\right],
$$

the covariance matrix of the measurements, which is a function of the actual distances $\rho_{i, k}$. The robot position uncertainty 
$\tilde{s}_{k}=\hat{s}_{k}-s_{k}$ derived from (5) has, hence, the following multilateration covariance matrix

$$
\Xi^{(n)}=\left(A^{(n)^{T}} N_{k}^{(n)^{-1}} A^{(n)}\right)^{-1}=\left[\begin{array}{cc}
\sigma_{x, k}^{2} & \sigma_{x y, k}^{2} \\
\sigma_{y x, k}^{2} & \sigma_{y y, k}^{2}
\end{array}\right],
$$

whose explicit form is reported in [40].

The problem presented in this paper is different from the classic multilateration just reported, since for the problem at hand, the positions of the anchors are deployed by the robot, hence affected by uncertainty except for the very first set $\mathcal{A}_{0}$. From this perspective, the problem is more similar to a Simultaneous Localisation And Mapping (SLAM) problem rather than a standard positioning problem. Indeed, while the ranging measurements from an anchor in $\mathcal{A}_{0}$ is simply (4), from the $i$-th deployed anchor turns to

$$
\bar{\rho}_{i, k}=\sqrt{\left(x_{k}-\hat{X}_{i}+\delta_{i_{x}}\right)^{2}+\left(y_{k}-\hat{Y}_{i}+\delta_{i_{y}}\right)^{2}}+\epsilon_{i, k},
$$

where we denote with $\delta_{i}=\left[\delta_{i_{x}}, \delta_{i_{y}}\right]^{T}$ the deployment error and with $\hat{a}_{i}=\left[\hat{X}_{i}, \hat{Y}_{i}\right]^{T}$ the estimated anchor position (i.e., $\left.a_{i}=\hat{a}_{i}-\delta_{i}\right)$. Assuming that the $i$-th anchor has been deployed at time $k T_{s}$, we have that $\hat{a}_{i}=\hat{s}_{k}$, hence given by (5), thus affected by an uncertainty described by the covariance matrix (7). In a typical SLAM problem, the first estimate of the position of a feature (which is used in the next steps as a landmark for localisation) is treated as the mean value of a random variable, usually considered as Gaussian. Applying this idea to the problem at hand, the feature estimate turns to be the anchor estimated position $\hat{a}_{i}$ and the $\delta_{i}$ the corresponding random variable of the uncertainty, assumed with zero-mean. To analyze the effect of the uncertainty, we may rewrite (8) with its first order Taylor approximation with respect to $\epsilon_{i, k}$ and $\delta_{i}$, thus obtaining

$$
\bar{\rho}_{i, k}=\rho_{i, k}+\epsilon_{i, k}+F \delta_{i}=\rho_{i, k}+\eta_{i, k},
$$

where $F=\frac{\partial \bar{\rho}_{i, k}}{\partial \delta_{i}}$ is the gradient of (8) evaluated in the mean value of $\delta_{i}$, i.e. $F$ is the same of $P$ in (3), but evaluated in $\hat{a}_{i}$. Therefore, using (9) instead of (4). the overall uncertainty for the ranging measurements from deployed anchors is expressed by $\eta_{i, k}$, which has zero-mean and variance

$$
\sigma_{\eta_{i, k}}^{2}=\sigma_{\rho}^{2}+F \Xi^{(n)} F^{T},
$$

where $\Xi^{(n)}$ is given in (7) (i.e., the robot position uncertainty during the placement). Since $\sigma_{\eta_{i, k}}^{2} \geq \sigma_{\rho}^{2}$, when the $i$-th deployed anchor is used, the ranging uncertainty will be larger. For instance, assuming that at time $k T_{s}$ the robot uses the anchors 1 and 2 from $\mathcal{A}_{0}$ and anchors $i$ and $j$ newly placed, i.e. for which only the estimates $\hat{a}_{i}$ and $\hat{a}_{j}$ are available, we have the new form of (6) as

$$
N_{k}^{(n)}=\left[\begin{array}{ccc}
\sigma_{\rho}^{2}\left(\rho_{1, k}^{2}+\rho_{2, k}^{2}\right) & \sigma_{\rho}^{2} \rho_{1, k}^{2} & \sigma_{\rho}^{2} \rho_{1, k}^{2} \\
\sigma_{\rho}^{2} \rho_{1, k}^{2} & \sigma_{\rho}^{2} \rho_{1, k}^{2}+\sigma_{\eta}^{2} \rho_{i, k} & \sigma_{\rho}^{2} \rho_{1, k}^{2} \\
\sigma_{\rho}^{2} \rho_{1, k}^{2} & \sigma_{\rho}^{2} \rho_{1, k}^{2} & \sigma_{\rho}^{2} \rho_{1, k}^{2}+\sigma_{\eta}^{2} \rho_{j, k}
\end{array}\right] .
$$

However, the previous development along the lines of the classic SLAM approach is not entirely correct, as empirically proved in Section V. Indeed, $\delta_{i}$ should not be considered as a random variable with zero-mean and covariance (7) but, instead, as a realization of a random variable at time $k T_{s}$,
TABLE I: Performance of the GANP algorithm versus parameter choices.

\begin{tabular}{|ccc|ccc|}
\hline \multicolumn{3}{|c|}{ Parameters } & \multicolumn{3}{c|}{ Performance indices } \\
\hline$w[\mathrm{~m}]$ & $r[\mathrm{~m}]$ & $n$ & $m$ & $d_{t}[\mathrm{~m}]$ & $c_{t}[\mathrm{~s}]$ \\
\hline 10 & 10 & 2 & 12 & 138 & 1118 \\
10 & 20 & 4 & 12 & 140 & 1007 \\
10 & 30 & 3 & 11 & 145 & 450 \\
30 & 10 & 4 & 10 & 166 & 307 \\
30 & 20 & 3 & 9 & 154 & 362 \\
30 & 30 & 2 & 9 & 146 & 895 \\
50 & 10 & 3 & 9 & 170 & 680 \\
50 & 20 & 2 & 9 & 180 & 650 \\
50 & 30 & 4 & 9 & 176 & 526 \\
\hline
\end{tabular}

i.e., a realization of the random variable modelling the robot positioning uncertainty, hence an unknown but constant offset. With this assumption, a typical non Bayesian approach as the nonlinear WLS can be adopted. More precisely, given at least three consecutive ranging measurements $\bar{\rho}_{i, k}, \bar{\rho}_{i, k+1}$ and $\bar{\rho}_{i, k+2}$ described in (8), the value of $\delta_{i}$ is given by

$$
\hat{\delta}_{i}=\underset{\left(\delta_{i_{x}}, \delta_{i_{y}}\right)}{\operatorname{argmin}} \sum_{j=k}^{k+2}\left[\left(\hat{x}_{j}-\hat{X}_{i}+\delta_{i_{x}}\right)^{2}+\left(\hat{y}_{j}-\hat{Y}_{i}+\delta_{i_{y}}\right)^{2}-\bar{\rho}_{i, j}^{2}\right]^{2} .
$$

This way, the offset $\delta_{i}$ induced in the anchor placement by the robot position uncertainty $\tilde{s}_{k}$ can be estimated and, hence, removed from the anchor estimated position $\hat{a}_{i}$ by means of this nonlinear unconstrained regression problem, as shown in the next section.

\section{SiMULATIONS AND EXPERIMENTS}

To evaluate the effectiveness of the GANP algorithm, we first present here the simulation results. We assume that the maximum ranging distance is $\rho^{m}=60 \mathrm{~m}$, which is derived by the hardware specification of the Decawave DWM1001 UWB anchors. To fine-tune the parameters of the GANP algorithm, i.e., the area width $w$, the number of subareas $n$ and the horizon of the prediction $r$, we report here an analysis based on the Taguchi Orthogonal Array (OA) design [41]. To this end, we impose the maximum PDoP value to be $p^{m}=1.5$ (a value guaranteeing low positioning uncertainty and a sufficiently large feasible placement region) and an exploration path length of approximately $60 \mathrm{~m}$. The result of the analysis, reported in terms of the performance indices number of anchors $m$, travelled distance $d_{t}$ and computational time $c_{t}$, is subsumed in Table I. It is evident that a larger area minimises the number of deployed anchors, since the feasible deploying space increases, at the price of a higher travelled distance. Instead, while the computation time clearly increases for larger areas (i.e., a larger space to explore for the GA algorithm), too small areas may imply difficulties in the search for a suitable solution. Hence the computation time increases as well. An optimal choice of the parameters would lead to the optimization of all the performance indices at once, which is hardly possible for the contrasting goals explained. Therefore, we compute the choice of the parameters $w, r$ and $n$ minimising each selected performance index at once and then compute the average among them, as reported in Table II. The placement obtained with the depicted tuning 
TABLE II: Optimal choices of the parameters.

\begin{tabular}{|c|ccc|}
\hline Performance indices & $w[\mathrm{~m}]$ & $r[\mathrm{~m}]$ & $n$ \\
\hline$m$ & 50 & 30 & 3 \\
$d_{t}$ & 10 & 30 & 2 \\
$c_{t}$ & 30 & 30 & 3 \\
\hline Average value & 27 & 30 & 3 \\
\hline
\end{tabular}

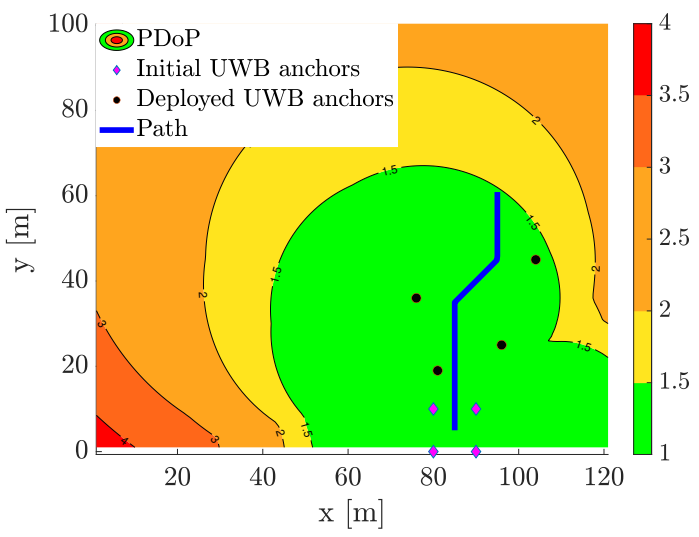

Fig. 6: PDoP level curves computed using $\mathcal{D}_{k}\left(s_{k}\right)$, where $s_{k}$ covers the entire map.

is then applied to a simulation example, thus obtaining the level curves of the PDoP reported in Figure 6. In this case, just 4 new anchors have been added to cover the entire exploration path and respecting the PDoP limit $p^{m}=1.5$. For comparison, Figure 7 reports the same scenario assuming the trivial approach sketched at the beginning of Section III. It is evident that, albeit simple, this algorithm implies a waste of resources, imposing the PDoP region $g\left(\mathcal{D}_{k}\left(s_{k}\right), s_{k}\right) \geq p^{m}$ to be too wide comapred to the exploration task. Moreover, as it can be observed from Figure 8, the PDoP constraint is not always verified along the exploration path or the placement path for the simple approach, while it is strictly satisfied for the GANP algorithm.

As a final simulation test, we verified that the SLAM-like assumption of the anchor estimated positions $\hat{a}_{i}$ cannot be considered as a random variable, as discussed in Section IV-B. To empirically prove this fact, we have carried out $10^{6}$ Monte Carlo trials where $\delta_{i}$ uncertainty is treated as a random variable contributing to the random, zero-mean white noise in (10) and hence applying the multilateration (5), which results in the position uncertainty in Figure 9, dashed line.

As can be noticed, this assumption end up with a nonnegligible bias on the estimates of the estimated position $\hat{s}_{k}$ (the Figure 9 reports the bias on the $\hat{x}_{k}$ axis, but it acts similarly on $\hat{y}_{k}$ ). Consequently, the bias should be treated as a constant but unknown quantity using (11), thus resulting in the unbiased estimation uncertainty of Figure 9, solid line. In the next section, this phenomenon is additionally highlighted in the experiments.

\section{A. Experimental results}

To test the algorithm on an actual set-up, we first characterise the UWB anchors at disposal. To this end, we carried out a Type A analysis [42], collecting at first repeated

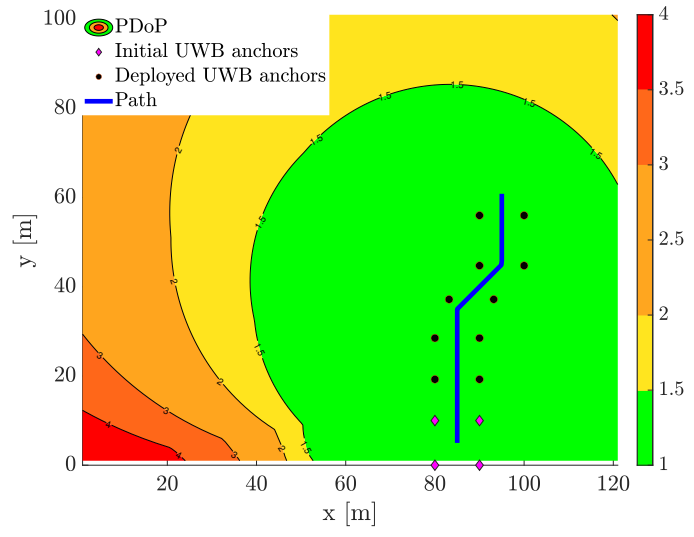

Fig. 7: PDoP level curves computed using $\mathcal{D}_{k}\left(s_{k}\right)$ for the straightforward algorithm sketched at the beginning of Section III.

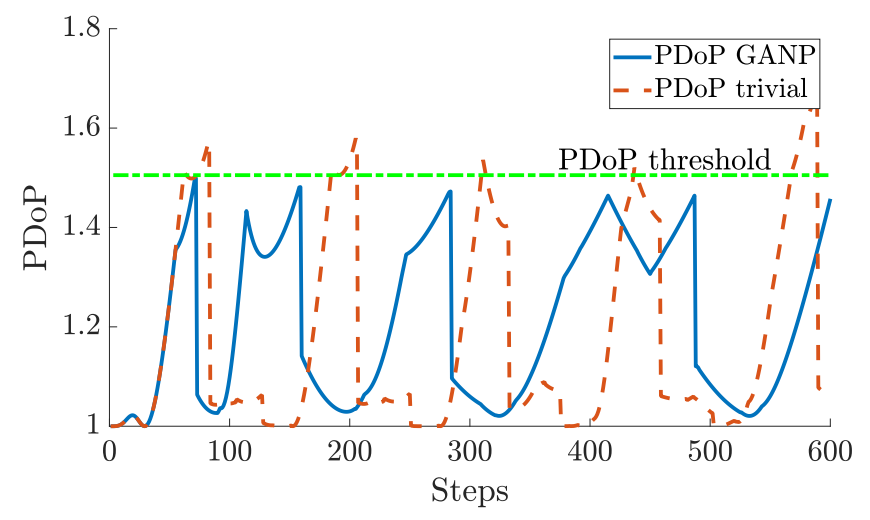

Fig. 8: PDoP evolution along the simulations in Figure 6 (GANP) and Figure 7 (trivial), respectively.

ranging measurements from known distances, i.e., at 1,3 and 7 meters. As an example, the histogram of the ranging measurements $\bar{\rho}_{i, k}$ in (4) collected from an UWB anchor Decawave DWM1001 at a distance of $3 \mathrm{~m}$ is reported for reference in Figure 10-a. Figure 10-b reports the histogram of the error on the position. Albeit all the available anchors behave similarly and with a relatively small variance $\sigma_{\rho}^{2}$, they all exhibit an approximately linear dependency on the actual distance $\rho_{i, k}$, as reported in Table III for the three sampled distances. We may noticed a slight increase of the bias and of the standard deviation $\sigma_{\rho}$, which can be compensated with a simple linear fitting model.

Since we do not have a large arena to test the system, we test the GANP placement algorithm forcing the anchors to be closed to each other by selecting $p^{m}=2$ to be above the minimum PDoP value obtained for the known first four anchors, which was $g\left(\mathcal{D}_{k}\left(s_{0}\right), s_{0}\right)=1.1\left(\mathcal{D}_{k}\left(s_{0}\right)=\left\{a_{1}, \ldots, a_{4}\right\}\right.$ in Figure 11). As stated previously, once the new anchor has been deployed in position $a_{5}$ and due to the positioning uncertainty of the UAV, the robot actually believes that the anchor is in $\hat{a}_{5}$ (Figure 11). After the placement, the UAV comes back to the exploration path (solid line in Figure 11) and, as described in Section IV-B, it stores three consecutive estimated positions, 


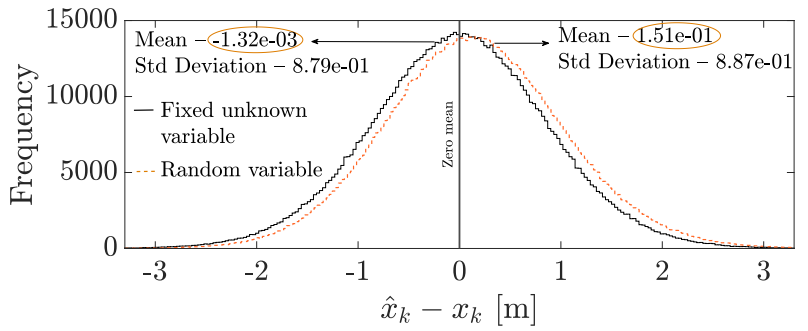

Fig. 9: Monte Carlo trials for the placement problem. When the placement error $\delta_{i}$ is treated as a random variable, a bias of about $15 \mathrm{~cm}$ is induced (dashed line), while if it is treated as an unknown but constant quantity estimated through (11) (solid line), the estimator is practically unbiased (bias around $1 \mathrm{~mm})$.

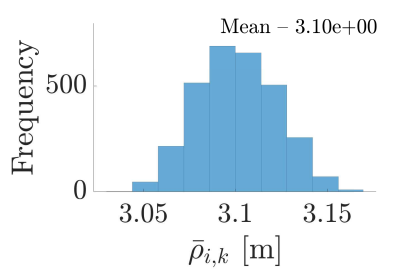

(a)

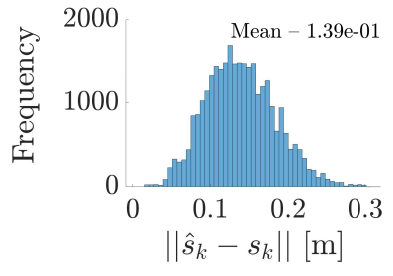

(b)
Fig. 10: (a) Characterisation of the ranging measurements $\bar{\rho}_{i, k}$ by means of an histogram obtained with 3000 consecutive measurements (b) Histogram of positioning error with 30000 consecutive measurements

namely $\hat{s}_{k}, \hat{s}_{k+1}$ and $\hat{s}_{k+2}$, which actually corresponds to the ideal values to be reached $s_{k}, s_{k+1}$ and $s_{k+2}$ (see Figure 11 for reference). To estimate the bias $\delta_{5}$, the robot collects 10 consecutive ranging measurements $\bar{\rho}_{5, k}$ from position $s_{k}$ and then compute the average. The process is then repeated from $s_{k+1}$ and $s_{k+2}$. The solution to (11) is then obtained using the Levenberg-Marquardt (LM) algorithm applied to the averages, thus obtaining the corrected location $a_{5}^{\text {correct }}$ of Figure 11, exhibiting a far reduced bias compared to $\hat{a}_{5}$. The method thus described has been compared with a linearized least square (LS) solution to (11) on such experimental data, which results in the comparison of Figure 12. Of course the iterative and incrementally precise approach of LM gives better results than LS for the bias estimation. Similarly, LM performs better of LS also for the standard positioning problem using multilateration. However, since the algorithm can be executed on board the vehicle and with constrained resources, the LM should be adopted with parsimony (its computation times is about 40 times compared to a linearized LS). As a consequence, we decided to keep the LM solution uniquely for the bias estimation problem.

\section{CONCLUSION}

This paper presented the development of a self-deployable UWB infrastructure by robots, with simulations and experiments done on small indoor UAVs to confirm the effectiveness of our approach. Starting from a minimal pre-deployed infrastructure, the robot can extend the UWB anchors geometry
TABLE III: Characterisation of bias and standard deviation of the ranging measurements

\begin{tabular}{|c|cc|}
\hline$\rho_{i, k}[\mathrm{~m}]$ & Bias $[\mathrm{m}]$ & $\sigma_{\rho}[\mathrm{m}]$ \\
\hline 1 & 0.06 & 0.029 \\
3 & 0.10 & 0.0231 \\
7 & 0.18 & 0.16 \\
\hline
\end{tabular}

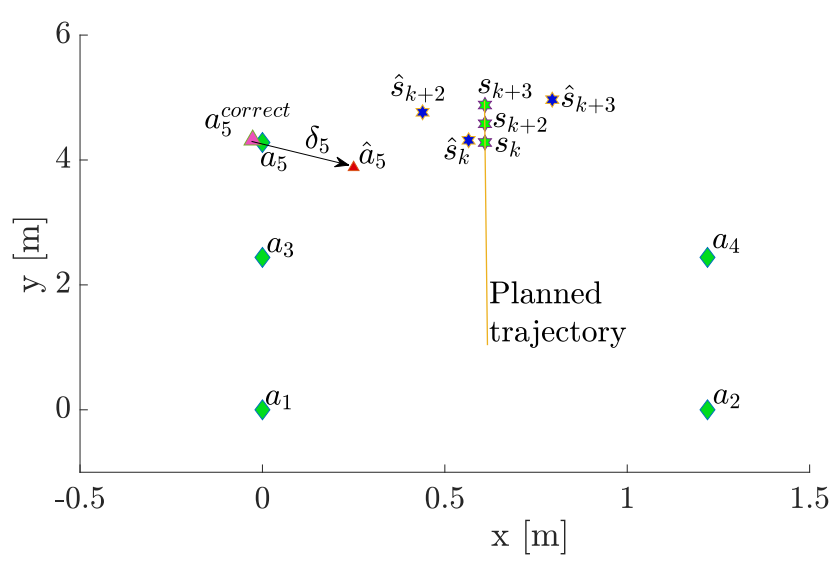

Fig. 11: Experimental results for the bias $\delta_{5}$ compensation.

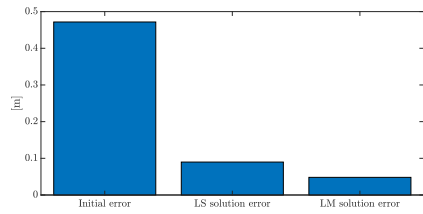

Fig. 12: Comparison between the LM and the LS solution to (11) with respect to the initial deployment error.

while exploring the environment during the mission. Clearly, uncertainty could increment while expanding the UWB infrastructure. To address this concern, we developed a genetic algorithm to compute the optimal placement of new anchors using the Geometric Dilution of Precision (GDoP). Simulation and experimental results demonstrated that the positioning algorithm uncertainty is always kept under the threshold required by the user. Future research threads will be devoted to apply the solution to a team of heterogeneous robots and to extend the analysis to the localisation problem, where the motion of the robot and its dynamic should be considered in the placement problem.

\section{REFERENCES}

[1] M. A. Rahman, S. Azad, A. T. Asyhari, M. Z. A. Bhuiyan, and K. Anwar, "Collab-sar: A collaborative avalanche search-and-rescue missions exploiting hostile alpine networks," IEEE Access, vol. 6, pp. 42 094-42 107, 2018.

[2] G. Seminara and D. Fontanelli, "First Responders Robotic Network for Disaster Management," in Modelling \& Simulation for Autonomous Systems (MESAS), J. Mazal, Ed. Cham: Springer International Publishing, 2018, pp. 350-373.

[3] D. St-Onge, M. Kaufmann, J. Panerati, B. Ramtoula, Y. Cao, E. B. J. Coffey, and G. Beltrame, "Planetary exploration with robot teams: Implementing higher autonomy with swarm intelligence," IEEE Robotics Automation Magazine, vol. 27, no. 2, pp. 159-168, 2020.

[4] Z. Dawei, Q. Lizhuang, Z. Demin, Z. Baohui, and G. Lianglin, "Unmanned aerial vehicle (uav) photogrammetry technology for dynamic mining subsidence monitoring and parameter inversion: A case study in china," IEEE Access, vol. 8, pp. 16372-16386, 2020. 
[5] T. He, Y. Zeng, and Z. Hu, "Research of multi-rotor uavs detailed autonomous inspection technology of transmission lines based on route planning," IEEE Access, vol. 7, pp. 114955-114965, 2019.

[6] M. Bacco, P. Barsocchi, P. Cassará, D. Germanese, A. Gotta, G. R. Leone, D. Moroni, M. A. Pascali, and M. Tampucci, "Monitoring ancient buildings: Real deployment of an iot system enhanced by uavs and virtual reality," IEEE Access, vol. 8, pp. 50 131-50 148, 2020.

[7] D. Murugan, A. Garg, and D. Singh, "Development of an adaptive approach for precision agriculture monitoring with drone and satellite data," IEEE Journal of Selected Topics in Applied Earth Observations and Remote Sensing, vol. 10, no. 12, pp. 5322-5328, 2017.

[8] M. William Díaz and J. José Cáceres, "A novel application of drones: thermal diagnosis of electrical and telecommunications infrastructure," in 2018 IEEE 38th Central America and Panama Convention (CONCAPAN XXXVIII), 2018, pp. 1-6.

[9] Y. Wang, G. Wei, Q. Guan, and Y. Liu, "A novel positioning system of uav based on ima-gps three-layer data fusion," IEEE Access, vol. 8, pp. $158449-158458,2020$.

[10] A. Carrio, J. Tordesillas, S. Vemprala, S. Saripalli, P. Campoy, and J. P. How, "Onboard detection and localization of drones using depth maps," IEEE Access, vol. 8, pp. 30480-30 490, 2020.

[11] O. De Silva, G. K. I. Mann, and R. G. Gosine, "An ultrasonic and vision-based relative positioning sensor for multirobot localization," IEEE Sensors Journal, vol. 15, no. 3, pp. 1716-1726, 2015

[12] Y. Lin, J. Hyyppä, and A. Jaakkola, "Mini-uav-borne lidar for fine-scale mapping," IEEE Geoscience and Remote Sensing Letters, vol. 8, no. 3, pp. 426-430, 2011.

[13] M. L. Rodríguez-Arévalo, J. Neira, and J. A. Castellanos, "On the importance of uncertainty representation in active slam," IEEE Transactions on Robotics, vol. 34, no. 3, pp. 829-834, 2018.

[14] B. Yang, L. Guo, R. Guo, M. Zhao, and T. Zhao, "A novel trilateration algorithm for rssi-based indoor localization," IEEE Sensors Journal, vol. 20, no. 14, pp. 8164-8172, 2020.

[15] V. Magnago, P. Corbalán, G. Picco, L. Palopoli, and D. Fontanelli, "Robot Localization via Odometry-assisted Ultra-wideband Ranging with Stochastic Guarantees," in Proc. IEEE/RSJ International Conference on Intelligent Robots and System (IROS). Macao, China: IEEE, Nov. 2019, pp. 1607-1613.

[16] M. Li, H. Zhu, S. You, and C. Tang, "Uwb-based localization system aided with inertial sensor for underground coal mine applications," IEEE Sensors Journal, vol. 20, no. 12, pp. 6652-6669, 2020.

[17] P. Nazemzadeh, D. Fontanelli, D. Macii, and L. Palopoli, "Indoor Localization of Mobile Robots through QR Code Detection and Dead Reckoning Data Fusion," IEEE/ASME Transactions on Mechatronics, vol. 22, no. 6, pp. 2588-2599, Dec. 2017.

[18] V. Magnago, L. Palopoli, A. Buffi, B. Tellini, A. Motroni, P. Nepa, D. Macii, and D. Fontanelli, "Ranging-free UHF-RFID Robot Positioning through Phase Measurements of Passive Tags," IEEE Trans. on Instrumentation and Measurement, vol. 69, no. 5, pp. 2408-2418, May 2020.

[19] V. Magnago, L. Palopoli, R. Passerone, D. Fontanelli, and D. Macii, "Effective Landmark Placement for Robot Indoor Localization with Position Uncertainty Constraints," IEEE Trans. on Instrumentation and Measurement, vol. 68, no. 11, pp. 4443-4455, November 2019.

[20] N. Bulusu, J. Heidemann, and D. Estrin, "Adaptive beacon placement," in Proceedings 21st International Conference on Distributed Computing Systems, 2001, pp. 489-498.

[21] G. Tuna, V. C. Gungor, and K. Gulez, "An autonomous wireless sensor network deployment system using mobile robots for human existence detection in case of disasters," Ad Hoc Networks, vol. 13, pp. 54 68, 2014, (1)Special Issue : Wireless Technologies for Humanitarian Relief and (2)Special Issue: Models And Algorithms For Wireless Mesh Networks.

[22] P. K. Yoon, S. Zihajehzadeh, B. Kang, and E. J. Park, "Robust biomechanical model-based 3-d indoor localization and tracking method using uwb and imu," IEEE Sensors Journal, vol. 17, no. 4, pp. 1084-1096, 2017.

[23] X. Yang, J. Wang, D. Song, B. Feng, and H. Ye, "A novel nlos error compensation method based imu for uwb indoor positioning system," IEEE Sensors Journal, pp. 1-1, 2021.

[24] S. Vandermeeren and H. Steendam, "Pdr/uwb based positioning of a shopping cart," IEEE Sensors Journal, pp. 1-1, 2021.

[25] W. Wang, D. Marelli, and M. Fu, "Multiple-vehicle localization using maximum likelihood kalman filtering and ultra-wideband signals," IEEE Sensors Journal, vol. 21, no. 4, pp. 4949-4956, 2021.

[26] M. Kolakowski, V. Djaja-Josko, and J. Kolakowski, "Static lidar assisted uwb anchor nodes localization," IEEE Sensors Journal, pp. 1-1, 2020.
[27] Q. Shi, S. Zhao, X. Cui, M. Lu, and M. Jia, "Anchor self-localization algorithm based on uwb ranging and inertial measurements," Tsinghua Science and Technology, vol. 24, no. 6, pp. 728-737, 2019.

[28] J. Díez-González, R. Álvarez, N. Prieto-Fernández, and H. Perez, "Local wireless sensor networks positioning reliability under sensor failure," Sensors, vol. 20, no. 5, 2020. [Online]. Available: https://www.mdpi.com/1424-8220/20/5/1426

[29] A. Darabseh, E. Bitsikas, B. Tedongmo, and C. Pöpper, "On ads-b sensor placement for secure wide-area multilateration," Proceedings, vol. 59, no. 1, 2020. [Online]. Available: https://www.mdpi.com/2504$3900 / 59 / 1 / 3$

[30] G. Feng, C. Shen, C. Long, and F. Dong, "GDOP index in UWB indoor location system experiment," in 2015 IEEE SENSORS, 2015, pp. 1-4.

[31] D. Fontanelli, "Perception for Autonomous Systems: A Measurement Perspective on Localisation and Positioning," Instrumentation and Measurement Magazine, 2021, to appear.

[32] Yang Yong and Miao Lingjuan, "Gdop results in all-in-view positioning and in four optimum satellites positioning with gps prn codes ranging," in PLANS 2004. Position Location and Navigation Symposium (IEEE Cat. No.04CH37556), 2004, pp. 723-727.

[33] I. Sharp, K. Yu, and Y. J. Guo, "Gdop analysis for positioning system design," IEEE Transactions on Vehicular Technology, vol. 58, no. 7, pp. 3371-3382, 2009

[34] C. Wu, W. Su, and Y. Ho, "A study on gps gdop approximation using support-vector machines," IEEE Transactions on Instrumentation and Measurement, vol. 60, no. 1, pp. 137-145, 2011.

[35] P. H. Dana, "Global positioning system (gps) time dissemination for real-time applications," Real-Time Systems, vol. 12, no. 1, pp. 9-40, Jan 1997. [Online]. Available: https://doi.org/10.1023/A:1007906014916

[36] R. J. MILLIKEN and C. J. ZOLLER, "Principle of operation of navstar and system characteristics," NAVIGATION, vol. 25, no. 2, pp. 95-106, 1978.

[37] F. Lazzari, A. Buffi, P. Nepa, and S. Lazzari, "Numerical investigation of an uwb localization technique for unmanned aerial vehicles in outdoor scenarios," IEEE Sensors Journal, vol. 17, no. 9, pp. 2896-2903, 2017.

[38] S. Karaman and E. Frazzoli, "Sampling-based algorithms for optimal motion planning," The international journal of robotics research, vol. 30, no. 7, pp. 846-894, 2011.

[39] M. Beinhofer, J. Müller, and W. Burgard, "Effective landmark placement for accurate and reliable mobile robot navigation," Robotics and Autonomous Systems, vol. 61, no. 10, pp. 1060-1069, 2013, selected Papers from the 5th European Conference on Mobile Robots (ECMR 2011). [Online]. Available: https://www.sciencedirect.com/science/article/pii/S0921889012001418

[40] D. Fontanelli, F. Shamsfakhr, D. Macii, and L. Palopoli, "An uncertaintydriven and observability-based state estimator for nonholonomic robots," IEEE Transactions on Instrumentation and Measurement, vol. 70, pp. $1-12,2021$.

[41] G. Taguchi, "Introduction to quality engineering: designing quality into products and processes," Tech. Rep., 1986.

[42] L. Kirkup and R. B. Frenkel, An introduction to uncertainty in measurement: using the GUM (guide to the expression of uncertainty in measurement). Cambridge University Press, 2006. 